\title{
Scattering cancellation by metamaterial cylindrical multilayers
}

\section{Simone Tricarico}

Filiberto Bilotti

bilotti@uniromaz.it

Lucio Vegni

\author{
Department of Applied Electronics, University "Roma Tre", Via della Vasca Navale, \\ 84 - 00146 Rome, Italy \\ Department of Applied Electronics, University "Roma Tre", Via della Vasca Navale, \\ 84 - 00146 Rome, Italy \\ Department of Applied Electronics, University "Roma Tre", Via della Vasca Navale, \\ 84 - 00146 Rome, Italy
}

In this paper, we present the theoretical analysis and the design of cylindrical multilayered electromagnetic cloaks based on the scattering cancellation technique. We propose at first the analysis and the design of bi-layered cylindrical shells, made of homogenous and isotropic metamaterials, in order to effectively reduce the scattered field from a dielectric cylindrical object. The single shell and the bi-layered shell cases are compared in terms of scattering reduction and loss effects. The comparison shows that the bi-layered configuration exhibits superior performances. The scattering cancellation approach, is, then, extended to the case of generic multilayered cylindrical shells, considering again homogeneous and isotropic metamaterials. The employment of the proposed technique to the case of cloaking devices working at multiple frequencies is also envisaged and discussed. Finally, some practical layouts of cylindrical electromagnetic cloaks working at optical frequencies are also proposed. In these configurations, the homogenous and isotropic metamaterials are replaced by their actual counterparts, obtained using alternating stacked plasmonic and non-plasmonic layers. The theoretical formulation and the design approaches presented throughout the paper are validated through proper full-wave numerical simulations. [D0I: 10.2971/je0s.2009.09021]

Keywords: electromagnetic cloaking, electromagnetic scattering, metamaterials

\section{INTRODUCTION}

Recently, the possibility to synthesize properly engineered cloaks of invisibility by employing metamaterials and plasmonic media has determined a growing interest in the study of transparency and scattering-free phenomena. Several groups worldwide have proposed alternative solutions [1][10] based on really different mechanisms, such as anomalous localized resonances [1] or coordinate transformations [2].

In particular, the approach relying on conformal mapping techniques is quite elegant and has led to some successful results, both at optical and microwave frequencies [3]-[5]. The main advantage of such cloaking devices is that they are mostly object independent [6]. This is because the approach results eventually in a rerouting of the electromagnetic radiation around the obstacle, effectively reducing the scattering outside the cloaked object. Some issues related to this approach concern the operative bandwidth, the losses, and the complex design of the covers, based on inhomogeneous and anisotropic materials. Such drawbacks limit the valuable cloaking effect to a narrow range of frequencies and to a given polarization.

An interesting alternative solution relies on the scattering cancellation technique proposed in [7]. The scattering cross section of a given object can be, in fact, drastically reduced by employing plasmonic and metamaterial cloaks, which can suppress the multi-polar scattering of relatively electrically large objects by exploiting the unusual properties of their local negative polarizability. This approach has the advantage of using homogeneous covers, reducing the complexity of the design, and is not based on intrinsically resonant phenomena, providing good performances in terms of bandwidth and response to geometric variations $[8,9]$ and losses. Since the covers must be inherently dispersive to obtain the desired combination of constitutive parameters, the cloaking effect is practically limited by the dispersion of the involved media.

At optical frequencies, noble metals exhibit already the required negative polarizability to enable the scattering cancellation [10]. However, such materials exhibit a strong plasmonic behavior and high losses at the visible frequencies. A negative polarizability may be obtained also using materials with close-to-zero values of the permittivity. Such materials, called Epsilon-Near-Zero (ENZ) metamaterials, show superior performances in terms of operational bandwidth and losses. However, the problem is that nature does not offer ENZ materials at visible frequencies and, thus, they have to be obtained artificially. One possibility has been presented in [11], where the authors have shown how the employment of alternating stacked plasmonic and non-plasmonic layers can be effectively used to synthesize the required permittivity profile and values at optical frequencies.

In this paper, we extend the scattering cancellation approach 
to the case of multi-layered cylindrical cloaks, which effectively reduce the scattering cross-section of cylindrical objects. A theoretical analysis of the structure is firstly presented, leading to the design formulas for multi-layered cylindrical cloaks. The actual implementation of the cylindrical shells working at optical frequencies is obtained using the approach already proposed in [11]. Finally, some results obtained through proper full-wave simulations are presented, in order to validate the proposed theoretical approach and to show its applicability also to the case of multi-frequency cloaking operation.

\section{THEORY AND DESIGN OF A BI-LAYERED CYLINDRICAL CLOAK}

Let's consider a time harmonic (the dependence $e^{j \omega t}$ is assumed in the paper) monochromatic plane wave with unit amplitude, which normally impinges on an infinitely long circular cylinder of radius $r_{1}$, with permittivity $\varepsilon_{1}$ and permeability $\mu_{1}$. We firstly assume that the cylinder is covered by two concentric homogeneous shells, with radii $r_{2}$ and $r_{3}$, and constitutive parameters $\left(\varepsilon_{2}, \mu_{2}\right)$ and $\left(\varepsilon_{3}, \mu_{3}\right)$, respectively (Figure 1$)$. The entire structure is surrounded by vacuum $\left(\varepsilon_{0}, \mu_{0}\right)$. We study the case of a $\mathrm{TM}_{z}$ polarized impinging wave, while the results for the $\mathrm{TE}_{z}$ polarization may be obtained by duality.
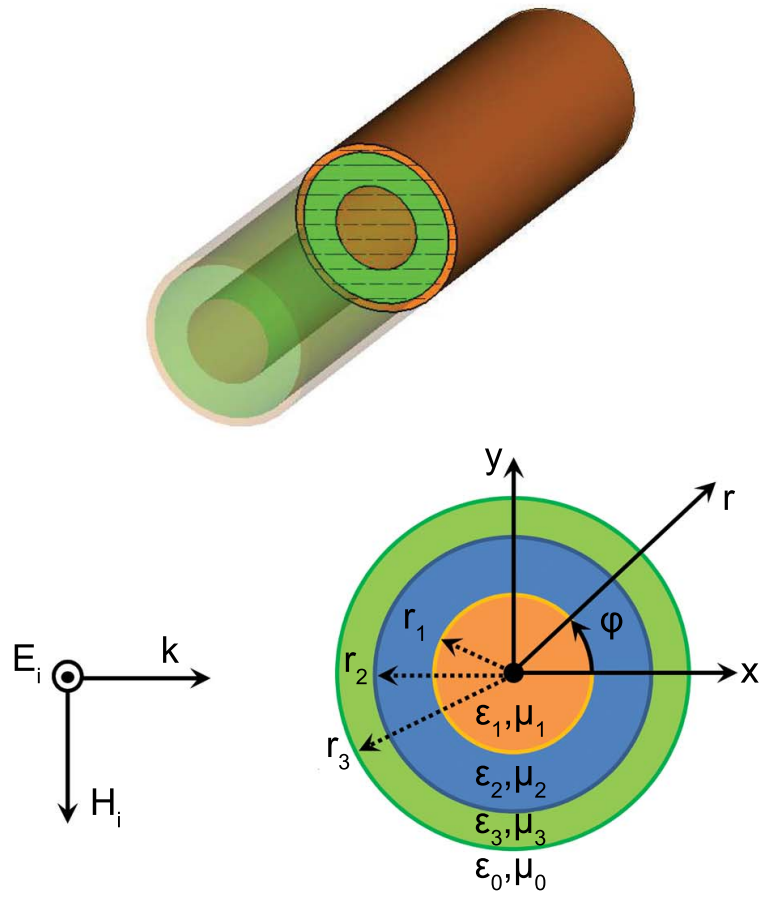

FIG. 1 TM polarized plane wave impinging on an infinitely long circular cylinder, covered with a bi-layered homogeneous shell.

We use a set of cylindrical coordinates $(r, \phi, z)$ and assume that the positive direction of propagation is along the $x$ axis $(\phi=0)$. Then, we perform a field expansion, expressing the incident electric field $\mathbf{E}_{i}$ as a superimposition of cylindrical waves [12]:

$$
\mathbf{E}^{i}=\hat{\mathbf{z}} E_{z}=\hat{\mathbf{z}} \sum_{n=0}^{\infty} j^{-n} \delta_{n} J_{n}\left(k_{0} r\right) \cos n \phi
$$

where $k_{0}=\omega \sqrt{\mu_{0} \varepsilon_{0}}$ is the free space wave number, $\delta_{n}$ the Neumann symbol and $J_{n}$ the Bessel function of the first kind and order $n$. The total field $\mathbf{E}$ outside the covered cylinder is given by the sum of $\mathbf{E}_{i}$ and scattered electric field $\mathbf{E}_{S}$, which can be consistently written as:

$$
\mathbf{E}=\mathbf{E}^{i}+\mathbf{E}^{\mathcal{S}}=\hat{\mathbf{z}} \sum_{n=0}^{\infty} j^{-n} \delta_{n}\left[J_{n}\left(k_{0} r\right)+c_{n} H_{n}^{(2)}\left(k_{0} r\right)\right] \cos n \phi,
$$

where $r \geq r_{3}, H_{n}^{(2)}$ is the Hankel function of the second kind of integer order $n$, and $c_{n}$ the unknown scattering coefficients.

The fields inside each cylindrical region can be factorized in terms of radial and azimuthal functions. The electric field in the cylindrical object can be, thus, expressed as:

$$
\mathbf{E}^{(1)}=\hat{\mathbf{z}} E_{z, 1}=\hat{\mathbf{z}} \sum_{n=0}^{\infty} j^{-n} \delta_{n} \ell_{n} J_{n}\left(k_{1} r\right) \cos n \phi, \quad 0 \leq r \leq r_{1}
$$

where $k_{1}$ is the wave number in the medium given by $k_{1}=\omega \sqrt{\mu_{1} \varepsilon_{1}}=k_{0} \sqrt{\mu_{r 1} \varepsilon_{r 1}}$, being $\left(\varepsilon_{r 1}, \mu_{r 1}\right)$ the relative permittivity and permeability of the material, respectively.

In the two cylindrical shells, the solution can be expressed as:

$$
\begin{aligned}
& \mathbf{E}^{(2)}=\hat{\mathbf{z}} E_{z}^{(2)} \\
& =\hat{\mathbf{z}} \sum_{n=0}^{\infty} j^{-n} \delta_{n}\left[a_{n} J_{n}\left(k_{2} r\right)+b_{n} Y_{n}\left(k_{2} r\right)\right] \cos n \phi, \quad r_{1} \leq r \leq r_{2} \\
& \mathbf{E}^{(3)}=\hat{\mathbf{z}} E_{z}^{(3)} \\
& =\hat{\mathbf{z}} \sum_{n=0}^{\infty} j^{-n} \delta_{n}\left[d_{n} J_{n}\left(k_{3} r\right)+f_{n} Y_{n}\left(k_{3} r\right)\right] \cos n \phi, \quad r_{2} \leq r \leq r_{3}
\end{aligned}
$$

where $k_{2}=\omega \sqrt{\mu_{2} \varepsilon_{2}}=k_{0} \sqrt{\mu_{r 2} \varepsilon_{r 2}}, k_{3}=\omega \sqrt{\mu_{3} \varepsilon_{3}}=k_{0} \sqrt{\mu_{r 3} \varepsilon_{r 3}}$, and $Y_{n}$ is the Bessel function of the second kind and order $n$. $a_{n}, b_{n}, d_{n}, f_{n}$ are unknown coefficients to be determined.

The magnetic field across all the structure may be obtained from the electric field by using Maxwell's equations. In particular, for the angular components of the magnetic field in the two shells, we obtain:

$$
\begin{aligned}
& H_{\phi}^{(2)}= \frac{k_{2}}{j \omega \mu_{2}} \sum_{n=0}^{\infty} j^{-n} \delta_{n}\left[a_{n} J_{n}^{\prime}\left(k_{2} r\right)+b_{n} Y_{n}^{\prime}\left(k_{2} r\right)\right] \cos n \phi, \\
& r_{1} \leq r \leq r_{2} \\
& H_{\phi}^{(3)}=\frac{k_{3}}{j \omega \mu_{2}} \sum_{n=0}^{\infty} j^{-n} \delta_{n}\left[d_{n} J_{n}^{\prime}\left(k_{3} r\right)+f_{n} Y_{n}^{\prime}\left(k_{3} r\right)\right] \cos n \phi,
\end{aligned}
$$$$
r_{2} \leq r \leq r_{3}
$$

Imposing the boundary conditions at the three interfaces:

$$
\begin{array}{ll}
\left.E_{z}^{(2)}\right|_{r=r_{1}}=\left.E_{z}^{(1)}\right|_{r=r_{1}}, & \left.H_{\phi}(3)\right|_{r=r_{2}}=\left.H_{\phi}^{(2)}\right|_{r=r_{2}} \\
\left.H_{\phi}^{(2)}\right|_{r=r_{1}}=\left.H_{\phi}^{(1)}\right|_{r=r_{1}}, & E_{z}^{i}+\left.E_{z}^{s}\right|_{r=r_{3}}=\left.E_{z}^{(3)}\right|_{r=r_{3}} \\
\left.E_{z}^{(3)}\right|_{r=r_{2}}=\left.E_{z}^{(2)}\right|_{r=r_{2}}, & H_{\phi}^{i}+\left.H_{\phi}^{s}\right|_{r=r_{3}}=\left.H_{\phi}^{(3)}\right|_{r=r_{3}}
\end{array}
$$

we can then calculate the vector of solutions $\mathbf{w}=$ $\left\{\ell_{n}, a_{n}, b_{n}, d_{n} f_{n}, c_{n}\right\}$. 
The previous relations can be written as a system in the form:

$$
\begin{aligned}
& a_{n} J_{n}\left(k_{2} r_{1}\right)+b_{n} Y_{n}\left(k_{2} r_{1}\right)=\ell_{n} J_{n}\left(k_{1} r_{1}\right) \\
& d_{n} J_{n}\left(k_{3} r_{2}\right)+f_{n} Y_{n}\left(k_{3} r_{2}\right)=a_{n} J_{n}\left(k_{2} r_{2}\right)+b_{n} Y_{n}\left(k_{2} r_{2}\right) \\
& J_{n}\left(k_{0} r_{3}\right)+c_{n} H_{n}^{(2)}\left(k_{0} r_{3}\right)=d_{n} J_{n}\left(k_{3} r_{3}\right)+f_{n} Y_{n}\left(k_{3} r_{3}\right) \\
& \frac{k_{2}}{\omega \mu_{2}}\left[a_{n} J_{n}^{\prime}\left(k_{2} r_{1}\right)+b_{n} Y_{n}^{\prime}\left(k_{2} r_{1}\right)\right]=\frac{k_{1}}{\omega \mu_{1}} \ell_{n} J_{n}^{\prime}\left(k_{1} r_{1}\right) \\
& \frac{k_{3}}{\omega \mu_{3}}\left[d_{n} J_{n}^{\prime}\left(k_{3} r_{2}\right)+f_{n} Y_{n}^{\prime}\left(k_{3} r_{2}\right)\right] \\
&=\frac{k_{2}}{\omega \mu_{2}}\left[a_{n} J_{n}^{\prime}\left(k_{2} r_{2}\right)+b_{n} Y_{n}^{\prime}\left(k_{2} r_{2}\right)\right] \\
& J_{n}^{\prime}\left(k_{0} r_{3}\right)+c_{n} H_{n}^{(2)},\left(k_{0} r_{3}\right) \\
&=\frac{k_{3}}{\omega \mu_{3}}\left[d_{n} J_{n}^{\prime}\left(k_{3} r_{3}\right)+f_{n} Y_{n}^{\prime}\left(k_{3} r_{3}\right)\right]
\end{aligned}
$$

or, in matrix form as $\mathbf{S} \cdot \mathbf{w}^{\mathbf{T}}=\mathbf{v}$, where $\mathbf{v}=$ $\left\{0,0,0,0, J_{n}\left(k_{0} r_{3}\right), J_{n}^{\prime}\left(k_{0} r_{3}\right)\right\}$ and $\mathbf{S}$ is the matrix of the system coefficients defined by Eq. (5).

The matrix $\mathbf{S}$ is certainly nonsingular, being the conditions (4) a set of linearly independent equations. In order to obtain the scattering coefficients $c_{n}$, we indicate with $\mathbf{S}_{c}$ the matrix formed by replacing the last column of $\mathbf{S}$ by the column vector $\mathbf{v}$. The scattering coefficients, thus, can be expressed as $c_{n}=\left|\mathbf{S}_{c}\right| /|\mathbf{S}|$, and, thus, are given by Eq. (6). If we choose $\varepsilon_{r 1}=\mu_{r 1}=\varepsilon_{r 2}=\mu_{r 2}=\varepsilon_{r 3}=\mu_{r 3}=1$ (free space propagation), then the $c_{n}$ vanish, being $\left|\mathbf{S}_{c}\right|$ zero (two columns are linearly dependent), while for $\varepsilon_{r 3}=\mu_{r 3}=1$ we reduce to the single cover case [13].

We can also express the scattering coefficients $c_{n}$ in a more convenient form, which will be useful in the next section, when we will extend this approach to multiple layers.

Remembering that $H_{n}^{(2)}=J_{n}-j Y_{n}$ and using the multi-linear properties of the determinants, we have:

$$
c_{n}=-\frac{B_{n}}{B_{n}+j A_{n}}
$$

where $B_{n}$ and $A_{n}$ are given by Eqs. (8) and (9) respectively. Using this form, which is similar to [13], it results that the zeroes of $c_{n}$ occur when $B_{n}=0$, being the denominator a finite quantity.

The total scattering cross section $(\sigma)$ of the structure can be expressed as [12]:

$$
\sigma=\frac{4}{k_{0}} \sum_{n=0}^{\infty} \delta_{n}\left|c_{n}\right|^{2}
$$

Let's consider first the case of an electrically thin cylindrical object covered by electrically thin shells $\left(k_{1} r_{1} \ll 1, k_{2} r_{2} \ll 1\right.$, $\left.k_{3} r_{3} \ll 1, k_{0} r_{3} \ll 1\right)$. In this case, according to [13] the $n=0$ order is the dominant one and the corresponding scattering coefficient, obtained from Eq. (11) when $n=0$, can be expressed using a series expansion as:

$$
\begin{aligned}
c_{0} \approx j \pi \frac{\left(k_{0} r_{1}\right)^{2}}{4}\left[\varepsilon_{r 1}+\left(\alpha^{2}-1\right) \varepsilon_{r 2}\right. & \left.+\beta^{2}\left(\varepsilon_{r 3}-1\right)-\alpha^{2} \varepsilon_{r 3}\right] \\
& +o\left(k_{0} r_{1}\right)^{4}
\end{aligned}
$$

where the positions $r_{2}=\alpha r_{1}$ and $r_{3}=\beta r_{1}$, being $\beta>\alpha>1$, have been used.

In order to minimize the scattering cross section of the cylindrical object, thus, this first order scattering coefficient $c_{0}$ does go to zero. This happens when the following condition holds:

$$
\varepsilon_{r 1}+\left(\alpha^{2}-1\right) \varepsilon_{r 2}+\beta^{2}\left(\varepsilon_{r 3}-1\right)-\alpha^{2} \varepsilon_{r 3}=0
$$

This means that the ratio $\alpha$ between the radius of the first shell and the radius of the object should match the relation:

$$
\alpha_{0}=\sqrt{\frac{\varepsilon_{r 2}-\varepsilon_{r 1}-\beta^{2}\left(\varepsilon_{r 3}-1\right)}{\varepsilon_{r 2}-\varepsilon_{r 3}}}
$$

while for the higher order terms $n=1,2, \ldots$ it can be proven that the relation becomes:

$\alpha_{n}=$

$\left[\frac{\left(\mu_{r 2}+\mu_{r 3}\right)\left[\beta^{2 n}\left(\mu_{r 3}-1\right)\left(\mu_{r 1}-\mu_{r 2}\right)+\left(\mu_{r 3}+1\right)\left(\mu_{r 1}+\mu_{r 2}\right)\right]+M_{n}}{2\left(\mu_{r 1}+\mu_{r 2}\right)\left(\mu_{r 2}-\mu_{r 3}\right)\left(\mu_{r 3}+1\right)}\right]^{\frac{1}{2 n}}$

where

$$
\begin{aligned}
& M_{n}=\left[4 \beta^{2 n}\left(\mu_{r 2}^{2}-\mu_{r 1}^{2}\right)\left(\mu_{r 2}-\mu_{r 3}\right)^{2}\left(\mu_{r 2}^{2}-1\right)+\right. \\
& \left.\left(\mu_{r 2}+\mu_{r 3}\right)^{2}\left\{\beta^{2 n}\left(\mu_{r 3}-1\right)\left(\mu_{r 2}+\mu_{r 1}\right)+\left(\mu_{r 3}+1\right)\left(\mu_{r 1}-\mu_{r 2}\right)\right\}\right]^{\frac{1}{2}}
\end{aligned}
$$

If the object has no magnetic properties, that is $\mu_{r 1}=\mu_{r 2}=\mu_{r 3}=1$, then the zero scattering condition for the generic order $n$, may be obtained when:

$$
\alpha_{n}=\sqrt[2 n+2]{\frac{\varepsilon_{r 2}-\varepsilon_{r 1}-\beta^{2 n+2}\left(\varepsilon_{r 3}-1\right)}{\varepsilon_{r 2}-\varepsilon_{r 3}}}, \quad n=0,1,2 \ldots
$$

Since the higher order scattering coefficients vanish at a higher rate (as shown in Figure 2), in the quasi static limit the condition Eq. (13) is sufficient to guarantee a sensible reduction of the scattering cross section of the cylindrical object.

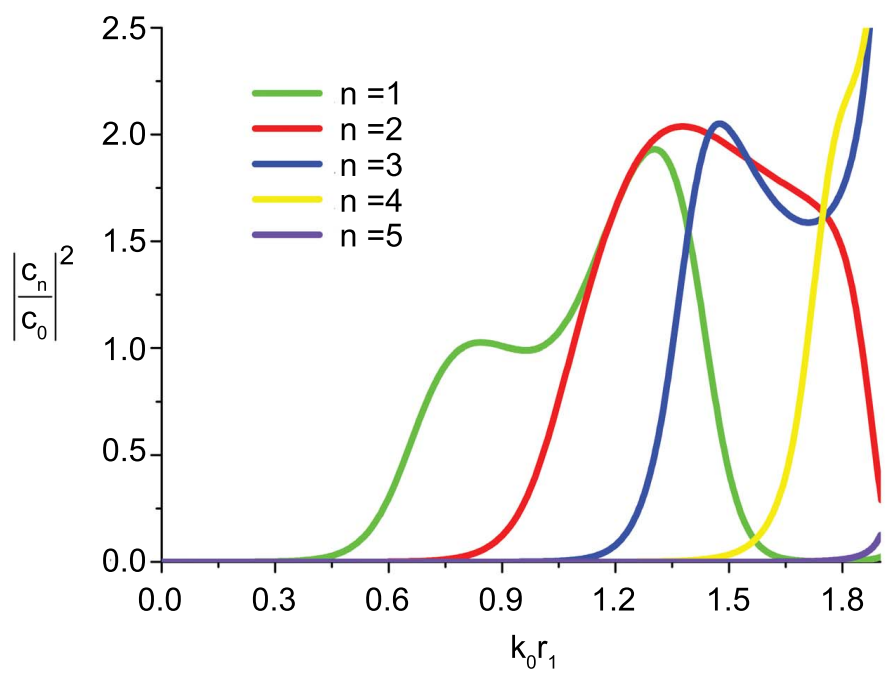

FIG. 2 Relative amplitude of the scattering coefficients of order $n>0$ for a TM polarized plane wave impinging on an infinitely long dielectric circular cylinder with $\varepsilon_{r 1}=4, \varepsilon_{r 2}=2, \varepsilon_{r 3}=3$ and $\alpha=1.5, \beta=2$. 


$$
\mathbf{S}=\left(\begin{array}{cccccc}
-J_{n}\left(k_{1} r_{1}\right) & J_{n}\left(k_{2} r_{1}\right) & Y_{n}\left(k_{2} r_{1}\right) & 0 & 0 & 0 \\
-\frac{k_{1}}{\omega \mu_{1}} J_{n}^{\prime}\left(k_{1} r_{1}\right) & \frac{k_{2}}{\omega \mu_{2}} J_{n}^{\prime}\left(k_{2} r_{1}\right) & \frac{k_{2}}{\omega \mu_{2}} Y_{n}^{\prime}\left(k_{2} r_{1}\right) & 0 & 0 & 0 \\
0 & J_{n}\left(k_{2} r_{2}\right) & Y_{n}\left(k_{2} r_{2}\right) & -J_{n}\left(k_{3} r_{2}\right) & -Y_{n}\left(k_{3} r_{2}\right) & 0 \\
0 & \frac{k_{2}}{\omega \mu_{2}} J_{n}^{\prime}\left(k_{2} r_{2}\right) & \frac{k_{2}}{\omega \mu_{2}} Y_{n}^{\prime}\left(k_{2} r_{2}\right) & -\frac{k_{3}}{\omega \mu_{3}} J_{n}^{\prime}\left(k_{3} r_{2}\right) & -\frac{k_{3}}{\omega \mu_{3}} Y_{n}^{\prime}\left(k_{3} r_{2}\right) & 0 \\
0 & 0 & 0 & J_{n}\left(k_{3} r_{3}\right) & Y_{n}\left(k_{3} r_{3}\right) & -H_{n}^{(2)}\left(k_{0} r_{3}\right) \\
0 & 0 & 0 & \frac{k_{3}}{\omega \mu_{3}} J_{n}^{\prime}\left(k_{3} r_{3}\right) & \frac{k_{3}}{\omega \mu_{3}} Y_{n}^{\prime}\left(k_{3} r_{3}\right) & -H_{n}^{(2)},\left(k_{0} r_{3}\right)
\end{array}\right)
$$

$$
c_{n}=-\frac{\left|\begin{array}{cccccc}
J_{n}\left(k_{1} r_{1}\right) & J_{n}\left(k_{2} r_{1}\right) & Y_{n}\left(k_{2} r_{1}\right) & 0 & 0 & 0 \\
\frac{k_{1}}{\omega \mu_{1}} J_{n}^{\prime}\left(k_{1} r_{1}\right) & \frac{k_{2}}{\omega \mu_{2}} J_{n}^{\prime}\left(k_{2} r_{1}\right) & \frac{k_{2}}{\omega \mu_{2}} Y_{n}^{\prime}\left(k_{2} r_{1}\right) & 0 & 0 & 0 \\
0 & J_{n}\left(k_{2} r_{2}\right) & Y_{n}\left(k_{2} r_{2}\right) & -J_{n}\left(k_{3} r_{2}\right) & -Y_{n}\left(k_{3} r_{2}\right) & 0 \\
0 & \frac{k_{2}}{\omega \mu_{2}} J_{n}^{\prime}\left(k_{2} r_{2}\right) & \frac{k_{2}}{\omega \mu_{2}} Y_{n}^{\prime}\left(k_{2} r_{2}\right) & -\frac{k_{3}}{\omega \mu_{3}} J_{n}^{\prime}\left(k_{3} r_{2}\right) & -\frac{k_{3}}{\omega \mu_{3}} Y_{n}^{\prime}\left(k_{3} r_{2}\right) & 0 \\
0 & 0 & 0 & J_{n}\left(k_{3} r_{3}\right) & Y_{n}\left(k_{3} r_{3}\right) & J_{n}\left(k_{0} r_{3}\right) \\
0 & 0 & 0 & \frac{k_{3}}{\omega \mu_{3}} J_{n}^{\prime}\left(k_{3} r_{3}\right) & \frac{k_{3}}{\omega \mu_{3}} Y_{n}^{\prime}\left(k_{3} r_{3}\right) & J_{n}^{\prime}\left(k_{0} r_{3}\right)
\end{array}\right|}{|\mathbf{S}|}
$$

$$
B_{n}=\left|\begin{array}{cccccc}
J_{n}\left(k_{1} r_{1}\right) & J_{n}\left(k_{2} r_{1}\right) & Y_{n}\left(k_{2} r_{1}\right) & 0 & 0 & 0 \\
\frac{k_{1}}{\omega \mu_{1}} J_{n}^{\prime}\left(k_{1} r_{1}\right) & \frac{k_{2}}{\omega \mu_{2}} J_{n}^{\prime}\left(k_{2} r_{1}\right) & \frac{k_{2}}{\omega \mu_{2}} Y_{n}^{\prime}\left(k_{2} r_{1}\right) & 0 & 0 & 0 \\
0 & J_{n}\left(k_{2} r_{2}\right) & Y_{n}\left(k_{2} r_{2}\right) & -J_{n}\left(k_{3} r_{2}\right) & -Y_{n}\left(k_{3} r_{2}\right) & 0 \\
0 & \frac{k_{2}}{\omega \mu_{2}} J_{n}^{\prime}\left(k_{2} r_{2}\right) & \frac{k_{2}}{\omega \mu_{2}} Y_{n}^{\prime}\left(k_{2} r_{2}\right) & -\frac{k_{3}}{\omega \mu_{3}} J_{n}^{\prime}\left(k_{3} r_{2}\right) & -\frac{k_{3}}{\omega \mu_{3}} Y_{n}^{\prime}\left(k_{3} r_{2}\right) & 0 \\
0 & 0 & 0 & J_{n}\left(k_{3} r_{3}\right) & Y_{n}\left(k_{3} r_{3}\right) & J_{n}\left(k_{0} r_{3}\right) \\
0 & 0 & 0 & \frac{k_{3}}{\omega \mu_{3}} J_{n}^{\prime}\left(k_{3} r_{3}\right) & \frac{k_{3}}{\omega \mu_{3}} Y_{n}^{\prime}\left(k_{3} r_{3}\right) & J_{n}^{\prime}\left(k_{0} r_{3}\right)
\end{array}\right|
$$

$$
A_{n}=\left|\begin{array}{cccccc}
J_{n}\left(k_{1} r_{1}\right) & J_{n}\left(k_{2} r_{1}\right) & Y_{n}\left(k_{2} r_{1}\right) & 0 & 0 & 0 \\
\frac{k_{1}}{\omega \mu_{1}} J_{n}^{\prime}\left(k_{1} r_{1}\right) & \frac{k_{2}}{\omega \mu_{2}} J_{n}^{\prime}\left(k_{2} r_{1}\right) & \frac{k_{2}}{\omega \mu_{2}} Y_{n}^{\prime}\left(k_{2} r_{1}\right) & 0 & 0 & 0 \\
0 & J_{n}\left(k_{2} r_{2}\right) & Y_{n}\left(k_{2} r_{2}\right) & -J_{n}\left(k_{3} r_{2}\right) & -Y_{n}\left(k_{3} r_{2}\right) & 0 \\
0 & \frac{k_{2}}{\omega \mu_{2}} J_{n}^{\prime}\left(k_{2} r_{2}\right) & \frac{k_{2}}{\omega \mu_{2}} Y_{n}^{\prime}\left(k_{2} r_{2}\right) & -\frac{k_{3}}{\omega \mu_{3}} J_{n}^{\prime}\left(k_{3} r_{2}\right) & -\frac{k_{3}}{\omega \mu_{3}} Y_{n}^{\prime}\left(k_{3} r_{2}\right) & 0 \\
0 & 0 & 0 & J_{n}\left(k_{3} r_{3}\right) & Y_{n}\left(k_{3} r_{3}\right) & -Y_{n}\left(k_{0} r_{3}\right) \\
0 & 0 & 0 & \frac{k_{3}}{\omega \mu_{3}} J_{n}^{\prime}\left(k_{3} r_{3}\right) & \frac{k_{3}}{\omega \mu_{3}} Y_{n}^{\prime}\left(k_{3} r_{3}\right) & -Y_{n}^{\prime}\left(k_{0} r_{3}\right)
\end{array}\right|
$$

Looking at Eq. (12), we can rearrange the cloak condition to give:

$$
\frac{\beta^{2}}{\gamma^{2}}+\frac{\alpha^{2}}{\eta^{2}}=1, \quad \text { where } \quad \frac{\varepsilon_{r 1}-\varepsilon_{r 2}}{1-\varepsilon_{r 3}}=\gamma^{2}, \quad \frac{\varepsilon_{r 1}-\varepsilon_{r 2}}{\varepsilon_{r 3}-\varepsilon_{r 2}}=\eta^{2}
$$

Only a given set of values of the electric parameters satisfies this relation. The equation, in fact, admits solutions only if the curve represented by Eq. (16) lies within the region delimited by the condition $\beta>\alpha>1$, depicted in Figure 3. Eq. (17) is a quadratic form that, according to the relative permittivity values $\varepsilon_{r 1}, \varepsilon_{r 2}, \varepsilon_{r 3}$, may represent in the plane $(\alpha, \beta)$ either a circle, or an ellipse, or an hyperbola.

\subsection{Case (a)}

When the electric parameters of the two covers are such that $\gamma^{2}=\eta^{2}=\zeta^{2}>0$ (i.e. when $2 \varepsilon_{r 3}=1+\varepsilon_{r 2}$ ), Eq. (17) is the equation of a circumference with radius $\zeta$. In this case, in order to have solutions of Eq. (16), it is not enough to assume $\zeta^{2}>0$, but it is necessary to assume $\zeta^{2}>2$, because the circle intersects the shaded solution region of Figure 4 only if $\zeta>\sqrt{2}$.

In the lossless case, thus, the possible choices of the electric pa-

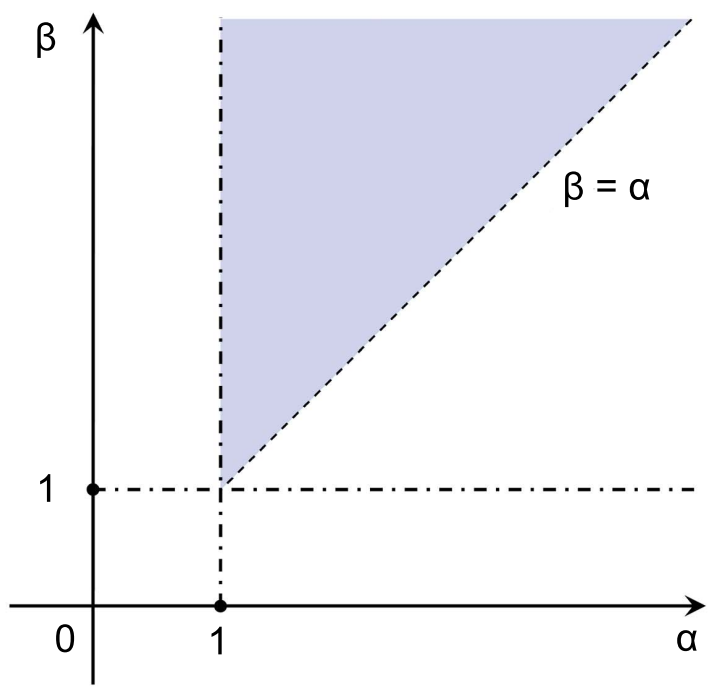

FIG. 3 Region of the $(\alpha, \beta)$ plane in which the zero scattering condition admits solutions.

rameters of the two cylindrical shells, when the permittivity of the object is given, may be summarized as shown in Figure 5.

It is clear from this flow chart that for an inner core filled with regular dielectric $\left(\varepsilon_{r 1}>1\right)$ only the use of metamaterials covers $\left(\varepsilon_{r 2}<1, \varepsilon_{r 3}<1\right)$ may lead to a suitable solution. How- 


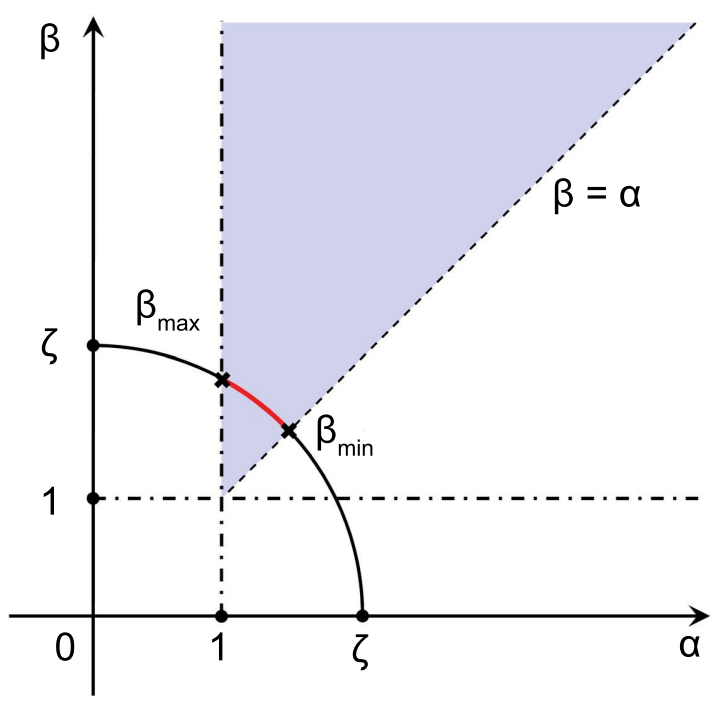

FIG. 4 Region of the $(\alpha, \beta)$ plane in which the zero scattering condition admits solutions when $\gamma^{2}=\eta^{2}=\zeta^{2}>2$.

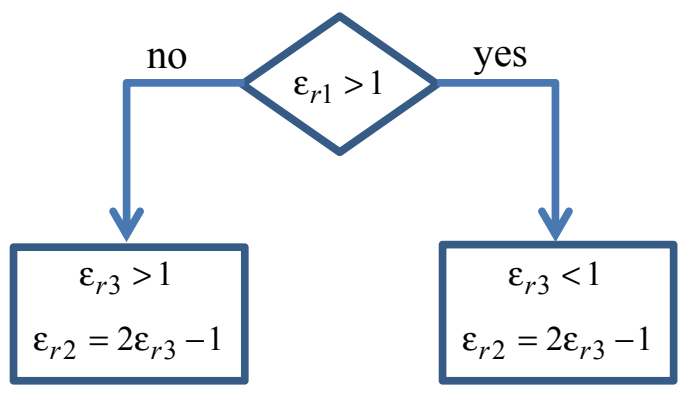

FIG. 5 Flow chart showing the possible choices of the electric parameters of the two cylindrical shells, when the permittivity of the object is given.

ever, not any arbitrary choice of the parameters according to may give solution to Eq. (17). In fact, only the portion of the circumference which lies in the shaded region of Figure 4 returns valid solutions. This further condition, allows to fix the values of the electric parameters and to choose the correct geometrical parameters of the two shells (i.e. the correct values of $\alpha$ and $\beta$ ). We note that moving along the circumference, the boundary values of $\beta$ for which we may have a solution are given by the intersections with the lines $\alpha=1\left(\beta_{\max }\right)$ and $\beta=\alpha\left(\beta_{\min }\right)$ as:

$$
\beta_{\min }^{2}=\frac{\varepsilon_{r 1}+1-2 \varepsilon_{r 3}}{2\left(1-\varepsilon_{r 3}\right)}, \quad \beta_{\max }^{2}=\frac{\varepsilon_{r 1}-\varepsilon_{r 3}}{1-\varepsilon_{r 3}} .
$$

Therefore, the choice of the radii of the shells should be such that:

$$
\frac{\varepsilon_{r 1}+1-2 \varepsilon_{r 3}}{2\left(1-\varepsilon_{r 2}\right)}<\beta^{2}<\frac{\varepsilon_{r 1}-\varepsilon_{r 3}}{1-\varepsilon_{r 3}}
$$

and

$$
\alpha^{2}=\zeta^{2}-\beta^{2}
$$

\subsection{Case (b)}

When both $\gamma^{2}>1, \eta^{2}>1$ and $\gamma^{2} \neq \eta^{2}$, Eq. (17) represents an ellipse, with the major axis oriented along $\alpha$ if $\gamma<\eta$, or along $\beta$ if $\gamma>\eta$. In this case, we may have two intersections,

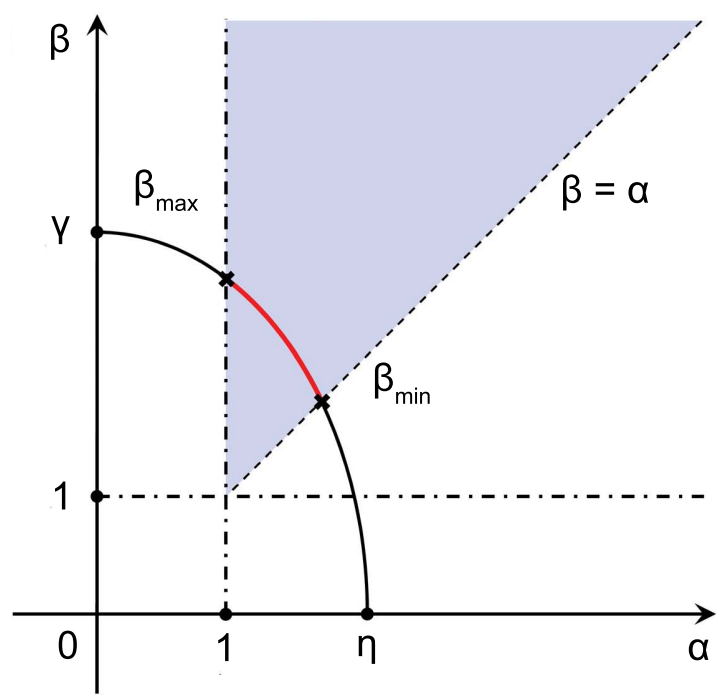

FIG. 6 Regions of the $(\alpha, \beta)$ plane in which the zero scattering condition admits solutions when $\gamma>1, \eta>1$, (case $\gamma>\eta$ ).

as depicted in Figure 6, at least if:

$$
\begin{aligned}
& \gamma^{2}=\frac{\varepsilon_{r 1}-\varepsilon_{r 2}}{1-\varepsilon_{r 3}}>1 \\
& \eta^{2}=\frac{\varepsilon_{r 1}-\varepsilon_{r 2}}{\varepsilon_{r 3}-\varepsilon_{r 2}}>1
\end{aligned}
$$

When both $(\gamma, \eta)>1$ it is, in fact, always possible to find two intersections for a given value of the eccentricity. If we choose, for instance, $\gamma>\eta$, then, the condition $\eta^{2}>1$ implies either $\varepsilon_{r 1}>\varepsilon_{r 3}>\varepsilon_{r 2}$ or $\varepsilon_{r 1}<\varepsilon_{r 3}<\varepsilon_{r 2}$. When either of these two conditions is verified, the ellipse intersects the lines delimiting the solution region and we obtain the values $\beta_{\min }$ and $\beta_{\max }$ as:

$$
\beta_{\min }^{2}=\frac{\varepsilon_{r 1}-\varepsilon_{r 2}}{1-\varepsilon_{r 2}}, \quad \beta_{\max }^{2}=\frac{\varepsilon_{r 1}-\varepsilon_{r 3}}{1-\varepsilon_{r 3}}
$$

By further assuming $\beta_{\text {max }}^{2}>1$, we have either $\varepsilon_{r 1}>1>\varepsilon_{r 3}$ or $\varepsilon_{r 1}<1<\varepsilon_{r 3}$. The latter conditions, combined with the previous ones derived from the condition $\eta^{2}>1$, determines the range of validity for the constitutive parameters of the object and of the shells to get the cloaking:

$$
\begin{aligned}
& \varepsilon_{r 1}>1>\varepsilon_{r 3}>\varepsilon_{r 2} \\
& \varepsilon_{r 1}<1<\varepsilon_{r 3}<\varepsilon_{r 2}
\end{aligned}
$$

Such quantities guarantee that both $\beta_{\min }^{2}>1$ and $\beta_{\text {max }}^{2}>$ 1 , being, obviously, $\beta_{\text {max }}^{2}>\beta_{\text {min }}^{2}$. Here again we see from Eqs. (22) that, in order to reduce the scattering contribution of an object made of a regular dielectric $\left(\varepsilon_{r 1}>1\right)$, we have to use shells with anomalous values (i.e. less than one) of the relative permittivity $\left(1>\varepsilon_{r 3}>\varepsilon_{r 2}\right)$. Finally, the radii of the two shells are to be chosen according to the following conditions:

$$
\begin{aligned}
\frac{\varepsilon_{r 1}-\varepsilon_{r 2}}{1-\varepsilon_{r 2}}<\beta^{2} & <\frac{\varepsilon_{r 1}-\varepsilon_{r 3}}{1-\varepsilon_{r 3}} \\
\frac{\beta^{2}}{\gamma^{2}}+\frac{\alpha^{2}}{\eta^{2}} & =1
\end{aligned}
$$

\subsection{Case (c)}

The last possible form for Eq. (17) occurs when either $\gamma^{2}$ or $\eta^{2}$ is a negative quantity. Looking at the expressions of $\gamma^{2}$ and 
$\eta^{2}$ involving the electrical parameters of the object and of the shells, in fact, both quantities cannot be negative at the same time. Moreover the electric parameters, which determine the sign of either $\gamma^{2}$ or $\eta^{2}$, inherently impose some limitations on the ranges of allowable values for such quantities. Let's consider, for instance, the case $\eta^{2}<0$, which is verified once either $\varepsilon_{r 1}<\varepsilon_{r 2}<\varepsilon_{r 3}$ or $\varepsilon_{r 1}>\varepsilon_{r 2}>\varepsilon_{r 3}$. In this case, Eq. (17) becomes:

$$
\frac{\beta^{2}}{\gamma^{2}}-\frac{\alpha^{2}}{\left|\eta^{2}\right|}=1
$$

Since we are interested only in a portion of the $(\alpha, \beta)$ plane, implying

$$
\beta_{\text {min }}^{2}>1 \Rightarrow \gamma^{2}>\frac{\left|\eta^{2}\right|}{1+\left|\eta^{2}\right|}
$$

in order to have solutions it should be at least $\gamma^{2} /\left|\eta^{2}\right|>1$. Only in this case, in fact, we may have one intersection $\beta_{\text {min }}^{2}>$ 1 with the line $\alpha=1$ :

$$
\beta_{\min }^{2}=\frac{\gamma^{2}}{\left|\eta^{2}\right|}\left(1+\left|\eta^{2}\right|\right)=\frac{\varepsilon_{r 1}-\varepsilon_{r 3}}{1-\varepsilon_{r 3}}>1
$$

(i.e. either $\varepsilon_{r 1}>1>\varepsilon_{r 3}$ or $\varepsilon_{r 1}<1<\varepsilon_{r 3}$ ), being the asymptote of the hyperbola $\beta=\sqrt{\gamma^{2} /\left|\eta^{2}\right|} \alpha$ within the region $\beta>\alpha>1$ (see Figure 7).

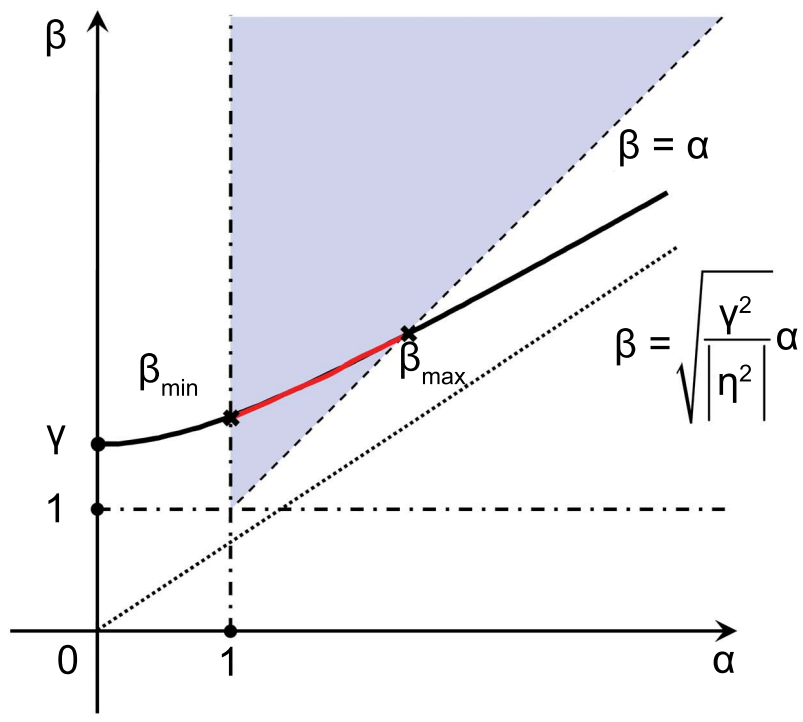

FIG. 7 Regions of the $(\alpha, \beta)$ plane in which the zero scattering condition admits solutions when $\eta^{2}<0$.

In this particular case, the design formulas for the two shells can be summarized as:

$$
\begin{gathered}
\varepsilon_{r 1}<1<\varepsilon_{r 3} \text { or } \varepsilon_{r 1}>1>\varepsilon_{r 3} \\
\frac{\varepsilon_{r 1}-\varepsilon_{r 3}}{1-\varepsilon_{r 3}}<\beta^{2}<\infty \\
\frac{\beta^{2}}{\gamma^{2}}-\frac{\alpha^{2}}{\left|\eta^{2}\right|}=1
\end{gathered}
$$

When the condition $0<\gamma^{2} /\left|\eta^{2}\right|<1$ is satisfied, that is for either $\varepsilon_{r 1}>1>\varepsilon_{r 2}>\varepsilon_{r 3}$ or $\varepsilon_{r 1}<1<\varepsilon_{r 2}<\varepsilon_{r 3}$, we have, instead, two possible intersections, not only $\beta_{\text {min }}$ (Eq. (25)), but also $\beta_{\max }$ :

$$
\beta_{\max }^{2}=\frac{\gamma^{2}\left|\eta^{2}\right|}{\left|\eta^{2}\right|-\gamma^{2}}=\frac{\varepsilon_{r 1}-\varepsilon_{r 2}}{1-\varepsilon_{r 2}}
$$

In this case, we have an upper limit for the values of $\beta$, and the design equations for the two shells are summarized as:

$$
\begin{gathered}
\varepsilon_{r 1}>1>\varepsilon_{r 2}>\varepsilon_{r 3} \quad \text { or } \varepsilon_{r 1}<1<\varepsilon_{r 2}<\varepsilon_{r 3} \\
\frac{\beta^{2}}{\gamma^{2}}-\frac{\alpha^{2}}{\left|\eta^{2}\right|}=1 \\
\frac{\varepsilon_{r 1}-\varepsilon_{r 3}}{1-\varepsilon_{r 3}}<\beta^{2}<\frac{\varepsilon_{r 1}-\varepsilon_{r 2}}{1-\varepsilon_{r 2}}
\end{gathered}
$$

Only if $\gamma^{2}<\left|\eta^{2}\right|\left(1+\left|\eta^{2}\right|\right)$ Eq. (24) does not admit solutions, since the entire curve does not intersect the region of interest.

To summarize, we may state that, given an object made of a regular dielectric $\left(\varepsilon_{r 1}>1\right)$, the choice of the effective parameters for the cover shells such that $\varepsilon_{r 3}<\varepsilon_{r 2}<1$, always leads to a suitable solution. These anomalous values of the relative permittivity can be achieved, thus, employing proper metamaterials with the required either negative or near-zero permittivity values.

Before concluding this sections, it is worth noticing that if in Eq. (17) it results that either $\gamma^{2} \rightarrow \infty$ or $\eta^{2} \rightarrow \infty$, the structure collapses into the one with one cover shell only and, thus, the solutions turns into those ones already presented in [13]. $\gamma^{2} \rightarrow \infty$, in fact, implies that $\varepsilon_{r 3}=1$, which means that the outer shell is removed. $\eta^{2} \rightarrow \infty$ implies, instead, that $\varepsilon_{r 3}=\varepsilon_{r 2}$, which means that the two shells merge into one.

\section{THEORY AND DESIGN OF A MULTI-LAYERED CYLINDRICAL CLOAK}

In this section, we extend the configuration to the case of $M$ cylindrical layers covering the cylindrical object. According to [14], we denote with the index $m=1,2, \ldots M$ the progressively stacked layers, being the surrounding vacuum symbolically represented as the $M+1$ "layer", extending to the entire outer space (see Figure 8). The procedure used in the previous section, based on the imposition of the boundary conditions of the field distributions Eq. (2), can be generalized to $M$ shell layers, by solving iteratively for the complex amplitude of the scattered field.

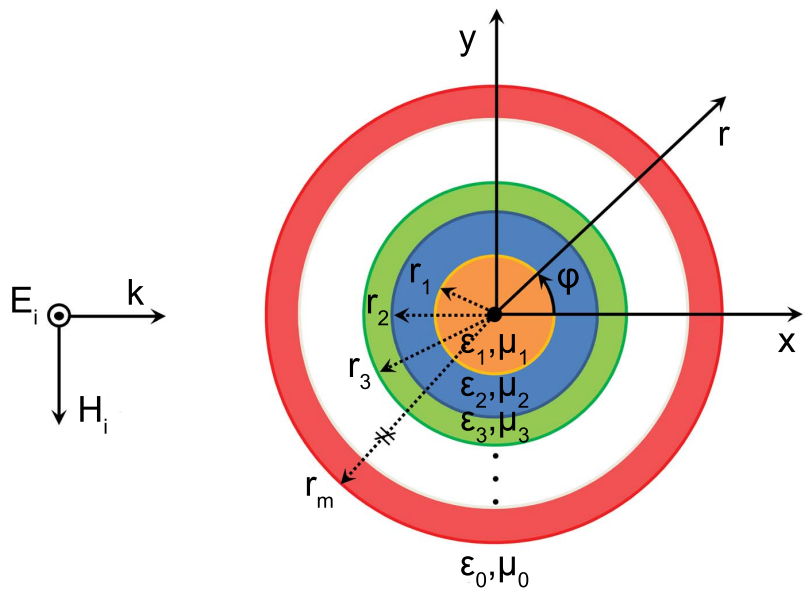

FIG. 8 TM-polarized plane wave impinging on an infinitely long circular cylinder, covered with a m-layered inhomogeneous shell. 
This approach is conveniently summarized in [14], where the following recursive formula is derived:

$$
\left(\begin{array}{c}
A_{m+1, n} \\
B_{m+1, n}
\end{array}\right)=\left(\begin{array}{ll}
U_{m n} & W_{m n} \\
V_{m n} & X_{m n}
\end{array}\right)\left(\begin{array}{c}
A_{m, n} \\
B_{m, n}
\end{array}\right)
$$

where:

$$
\begin{gathered}
U_{m n}=\mu_{m} k_{m+1} J_{n}\left(k_{m} r_{m}\right) Y_{n}^{\prime}\left(k_{m+1} r_{m}\right) \\
-\mu_{m+1} k_{m} J_{n}^{\prime}\left(k_{m} r_{m}\right) Y_{n}\left(k_{m+1} r_{m}\right) \\
V_{m n}=\mu_{m+1} k_{m} J_{n}\left(k_{m+1} r_{m}\right) J_{n}^{\prime}\left(k_{m} r_{m}\right) \\
\quad-\mu_{m} k_{m+1} J_{n}^{\prime}\left(k_{m+1} r_{m}\right) J_{n}\left(k_{m} r_{m}\right) \\
W_{m n}=\mu_{m} k_{m+1} Y_{n}\left(k_{m} r_{m}\right) Y_{n}^{\prime}\left(k_{m+1} r_{m}\right) \\
-\mu_{m+1} k_{m} Y_{n}^{\prime}\left(k_{m} r_{m}\right) Y_{n}\left(k_{m+1} r_{m}\right) \\
X_{m n}=\mu_{m+1} k_{m} Y_{n}^{\prime}\left(k_{m} r_{m}\right) J_{n}\left(k_{m+1} r_{m}\right) \\
-\mu_{m} k_{m+1} Y_{n}\left(k_{m} r_{m}\right) J_{n}^{\prime}\left(k_{m+1} r_{m}\right)
\end{gathered}
$$

and $k_{m}=\omega \sqrt{\mu_{m} \varepsilon_{m}}=k_{0} \sqrt{\mu_{r m} \varepsilon_{r m}}$. The amplitude of the scattering coefficients is related to Eq. (30) by the equivalent of Eq. (7), as:

$$
c_{n}=-\frac{B_{M+1, n}}{B_{M+1, n}+j A_{M+1, n}}
$$

The first entries in the recursion formula may be assumed as:

$$
A_{1, n}=1, \quad B_{1, n}=0,
$$

eventually introducing a normalization factor to match the incident wave in the case of a non-unit amplitude. Starting from Eqs. (31), we may perform the same series expansion we have already done in the bi-layered cover. Assuming the overall thickness to be electrically small $\left(k_{1} r_{1} \ll 1, \ldots k_{m} r_{m} \ll 1, k_{0} r_{m} \ll 1\right)$, and considering only the dipolar term for non-magnetic objects, we may rewrite Eqs. (31) as:

$$
\begin{aligned}
U_{m 0} & \approx \frac{2 k_{0} \mu_{0}}{\pi k_{0} r_{m}}+o\left(k_{0} r_{m}\right) \\
V_{m 0} & \approx \frac{1}{2} k_{0}^{2} \mu_{0} r_{m}\left(\varepsilon_{r m+1}-\varepsilon_{r m}\right)+o\left(k_{0} r_{m}\right)^{3} \\
W_{m 0} & \approx \frac{2 k_{0} \mu_{0}}{\pi^{2} k_{0} r_{m}} \log \frac{\varepsilon_{r m}}{\varepsilon_{r m 1}}+o\left(k_{0} r_{m}\right) \\
X_{m 0} & \approx \frac{2 k_{0} \mu_{0}}{\pi k_{0} r_{m}}+o\left(k_{0} r_{m}\right)
\end{aligned}
$$

Substituting Eqs. (34) in Eq. (30) one can get a straight iterative rule to easy design multi-layered cylindrical covers. It is straightforward to verify that in the case of a bi-layered cover the recursion procedure here outlined returns exactly Eq. (15) obtained previously. returns exactly obtained previously. During the recursion procedure, one may neglect the terms which are infinitesimal of higher order, but obviously increasing the numbers of layers it is more difficult to satisfy the quasi static approximation, because the global thickness of the structure progressively get electrically larger. Nevertheless, following the results presented in Figure 8, Eqs. (34) may be considered as accurate in a certain range of electrical thicknesses of the shells. In fact, even if the proposed closed formulas have been obtained in the case of electrically small objects, the dipolar term used to describe the scattering behavior of the structure is a valid approximation even for relatively electrically larger object. This approach may be, indeed, also extended to a more general case. It is, in fact, always possible to consider a finite set of scattering coefficients from Eq. (10), and impose numerically that they shall vanish at the desired design frequency. This can be done by truncating the scattering series at a sufficiently high order and minimizing it, as also suggested in [7]. In this case, the scattering cancellation condition can be easily obtained with a multi-layered structure, employing materials with suitable electric parameters, retrieved by a numerical optimization procedure.
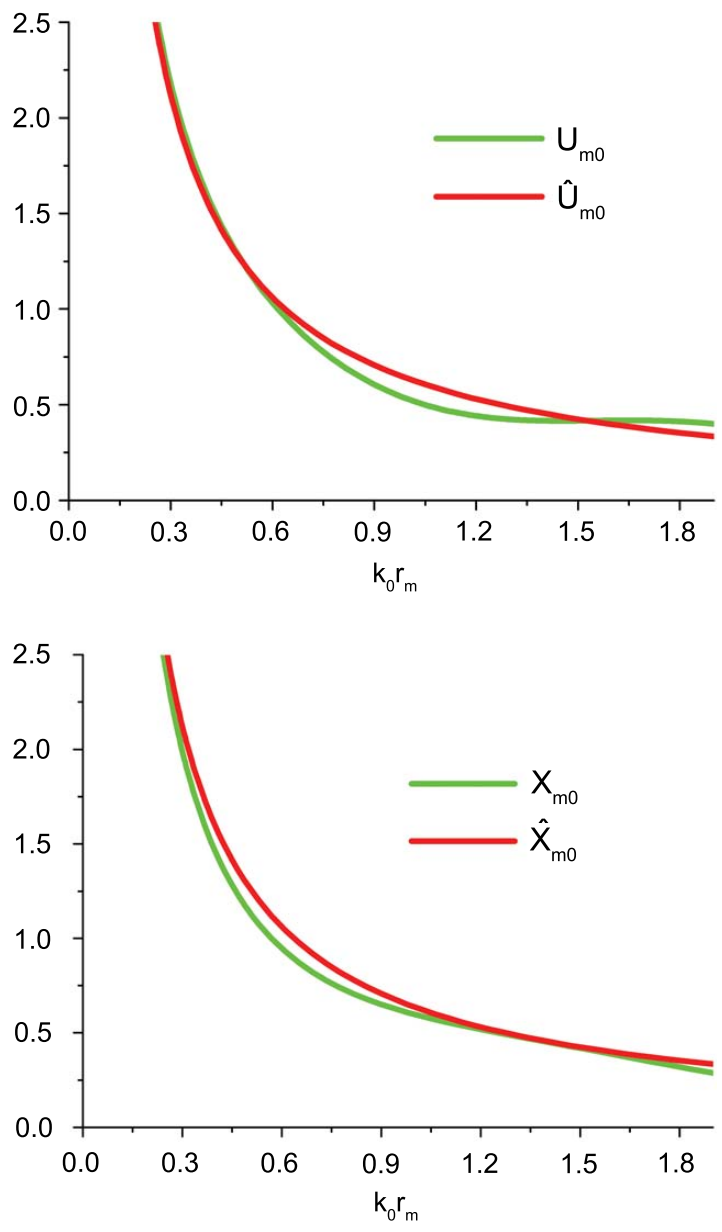

FIG. 9 Amplitude of the exact recursion matrix coefficients $U_{m 0}, X_{m 0}$ and of the approximated ones $\hat{U}_{m 0}, \hat{X}_{m 0}$ for two successive layers with dielectric contrast $\varepsilon_{r m+1}-\varepsilon_{r m}=1$.

\section{NUMERICAL RESULTS}

In order to validate the theoretical analysis for the multilayered cylindrical cloaking structures presented so far, we performed a set of numerical full-wave simulations through a commercial software based on the Finite Integration Technique. In the simulations we have taken into account some real life aspects, such as material losses, and compared them to the ideal results obtained in the previous sections.

Since we are interested in a configuration working at optical frequencies, we firstly considered a dielectric cylindrical object of finite length $L=500 \mathrm{~nm}$ and radius $r_{1}=30 \mathrm{~nm}$ made of silica $\left(\mathrm{SiO}_{2}\right)$ and illuminated by a monochromatic plane wave at the frequency of $600 \mathrm{THz}$. Since the far field scattering 
cross section of a finite cylinder is, in first approximation, proportional through its effective length $L^{2} / \lambda$ to Eq. (10), which refers to an infinitely long cylinder [12], the global procedure adopted in the previous sections remains applicable.

The outer shell is chosen to be made of silica, as well, with $\beta=2$. Using the design formulas presented throughout the paper, we got for $\alpha=1.8$ and $\varepsilon_{r 2} \approx-0.06$. In order to get a metamaterial shell exhibiting a near-zero permittivity in the visible, we refer to our previous work [11], where we illustrated how alternating layers of stacked plasmonic materials and regular dielectrics it is possible to implement homogeneous covers for a given TM polarization.

Following the aforementioned approach, we designed an ENZ cover based on alternating annular sections of silver (Ag) and silica. We, then, computed the scattering cross section and compared it with the one of the bare cylinder. As it can be seen from Figure 10, at $600 \mathrm{THz}$ we obtain a sensible reduction of the scattering width, implying a reduction of the object observability at and around the desired frequency.

It is worth noticing that, even though we have considered ma-

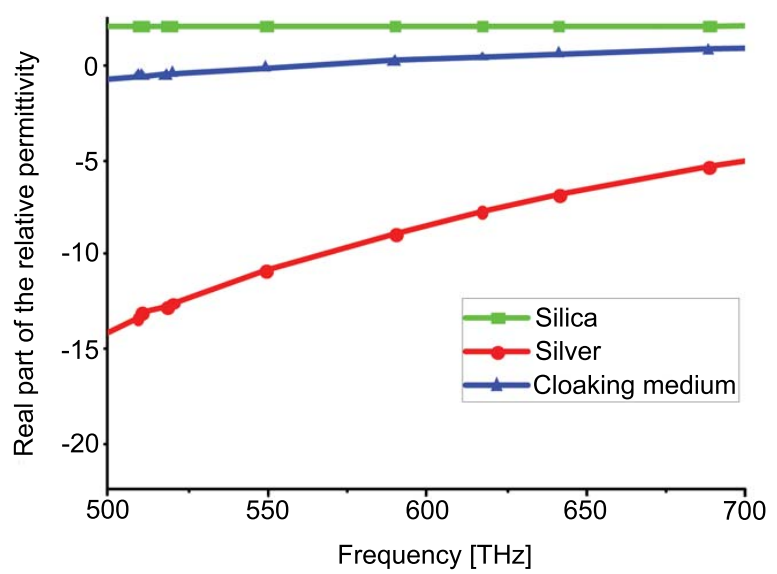

(a)

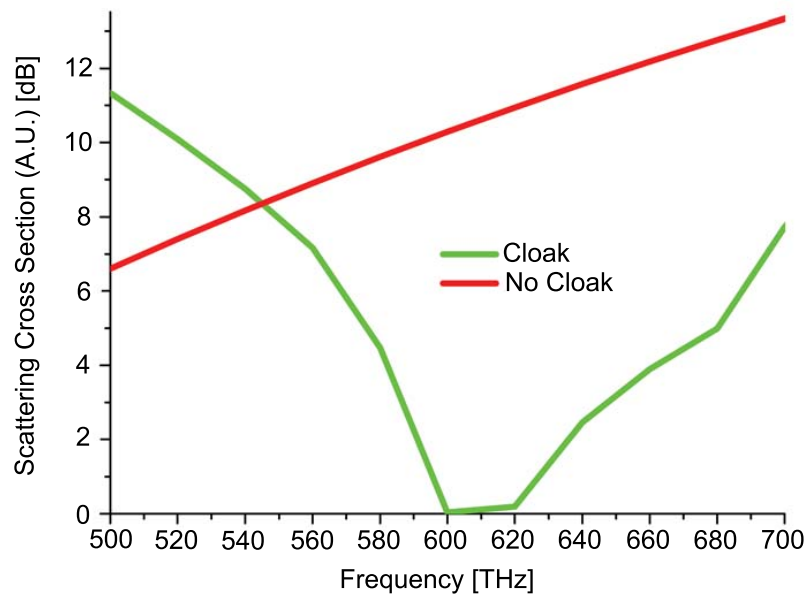

(b)

FIG. 10 (a) Real part of the permittivity of $\mathrm{Ag}, \mathrm{SiO}_{2}$ and of the cloak effective medium (component obtained along the axis of the cylinder for the TM polarization). The cloak is designed for $f=600 \mathrm{THz}$ and the thicknesses of the two material ( $\mathrm{Ag}$ and $\mathrm{SiO}_{2}$ ) shells are, $2 \mathrm{~nm}$ and $8 \mathrm{~nm}$, respectively. (b) Maximum of the scattering cross-section of a cylindrical object with and without the cloak.

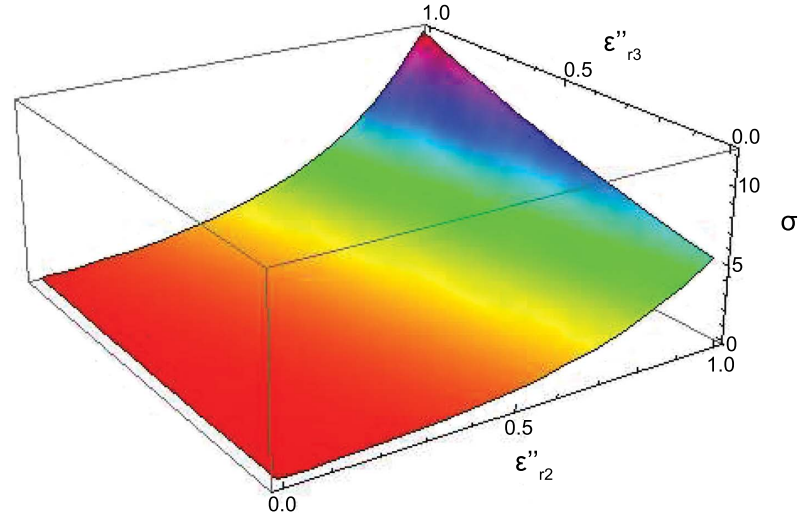

FIG. 11 Variation from the ideal (i.e. in the lossless case) total scattering cross section of a cylindrical object covered with a bi-layered shell made of lossy, homogenous materials, as a function of the imaginary parts of the relative permittivities of the layers. The real part of the permittivity is kept at the design value and the frequency is $f=600 \mathrm{THz}$.

terial losses in the simulations, especially due to the presence of a strong plasmonic material like silver, the scattering reduction is still quite evident. The robustness of this setup to losses is rather good, especially if compared with the performances of a single cover configuration [13]. The plot of the total scattering cross section of the cloaked cylinder as a function of the imaginary parts of the relative permittivities $\varepsilon_{r 2}^{\prime \prime}$ and $\varepsilon_{r 3}^{\prime \prime}$ of the two shells, is shown in Figure 11. This plot reveals that the scattering cancellation condition is valid also for a wide range of material losses, which is typical of several real-life layered materials.

In order to validate these results, we report also the distribution of the electric and magnetic field (magnitude and phase) for the bare cylinder and for the cloaked one (see Figures 1214). The transparency condition can be then justified by the appreciable shadow effect reduction we get for the cloaked cylinder.

The field lines, which are deformed by the scattering from the object in the case of the bare cylinder, are restored to the ones of a plane wave passing through the structure in the case of the cylindrical object covered by the cloak. This result is highlighted by the phase distributions shown in Figure 14.

An interesting aspect related to the employment of multilayered cover relies in the possibility to obtain a cloak effect at multiple frequencies, exploiting the dispersion profile of the involved materials, as done in [10] for the case of spherical shells. Looking at Eq. (12), in fact, one may obtain the scattering cancellation condition at different frequencies, for the same values of the radial extension $(\alpha, \beta)$ of the covers.

This can be achieved exploiting the new degrees of freedom introduced by the variation of the relative permittivities of the layers. However, for this geometry it is straightforward to verify from direct inspection of Eq. (13), that it is not trivial to design such covers with only Drude-like dispersive materials. A possible solution is to use also Lorentz dispersive media, in order to synthesize covers working at two different frequencies. 


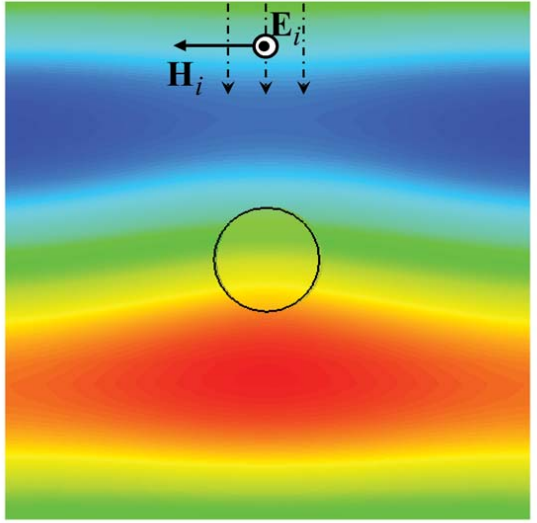

Bare Cylinder
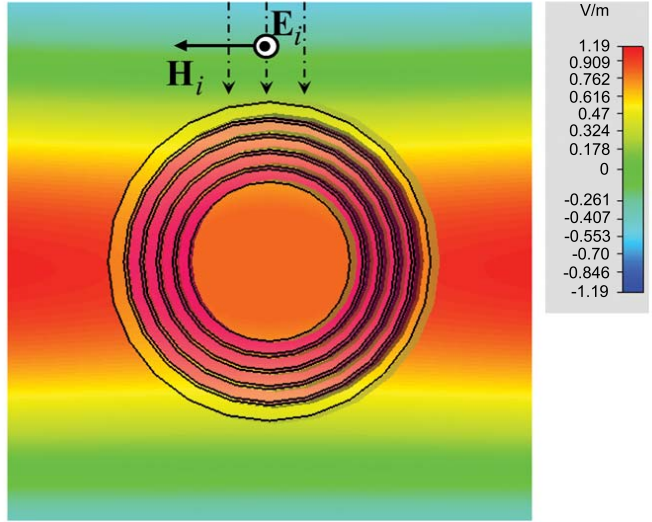

FIG. 12 Electric field distribution at the cloak frequency $f=600 \mathrm{THz}$.
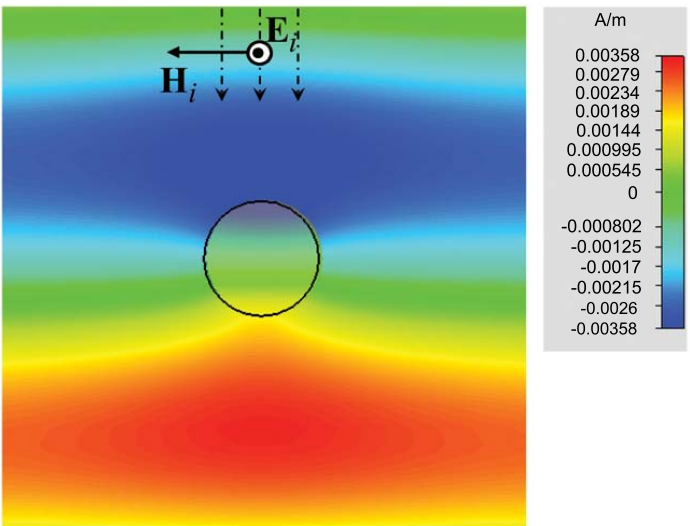

\section{Bare Cylinder}

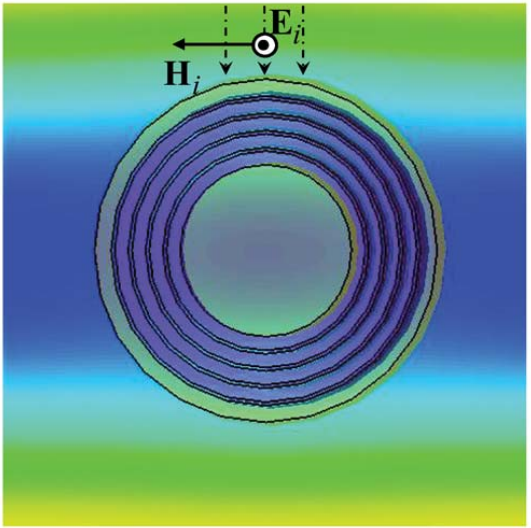

Cloaked Cylinder

FIG. 13 Magnetic field distribution at the cloak frequency $f=600 \mathrm{THz}$

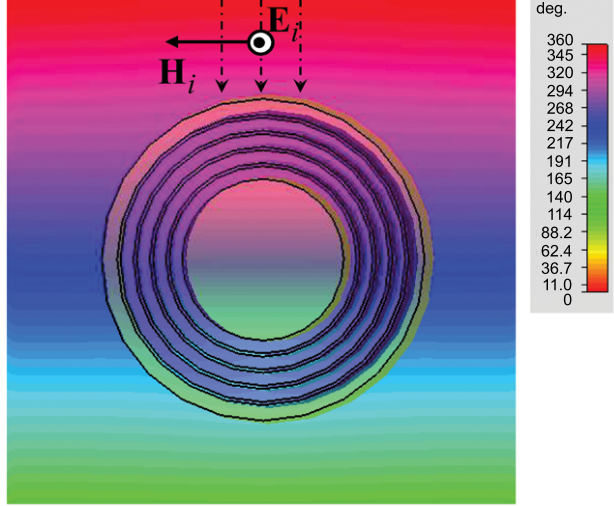

(a)
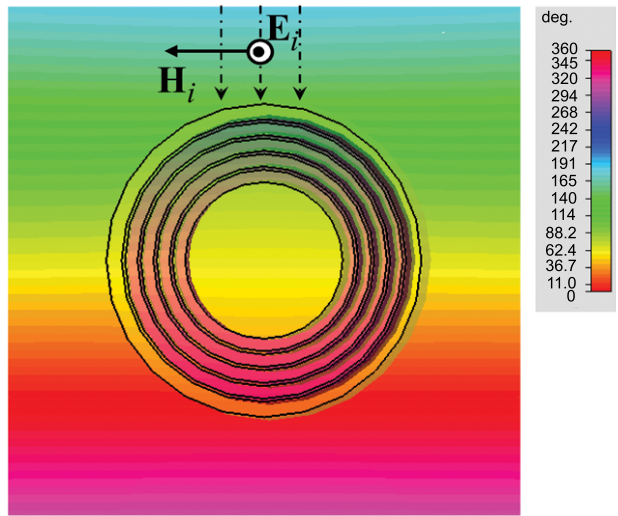

(b)

FIG. 14 Phase distribution of the electric (a) and magnetic (b) field for the covered structure at the cloak frequency $f=600 \mathrm{THz}$.

Just as an example of the new design capabilities enabled by multi-layered cloaks in the frame of multi-frequency operation, we consider again a cylinder made of silica, covered with a double layered shell with $\alpha=1.6$ and $\beta=2.6$. The outer shell is made of silver (see Figure 10(a) for silver dispersion), while the medium surrounding the inner core is a Lorentzlike dispersive material with resonant frequency of $600 \mathrm{THz}$ and collision frequency of $1 \mathrm{radTHz}$. Using the same design formulas of the previous cases, we are able to design the profile of $\varepsilon_{r 2}(\omega)$ in order to get the scattering cancellation at the two distinct frequencies $f_{1}=550 \mathrm{THz}$ and $f_{2}=650 \mathrm{THz}$. The graph reported in Figure 15 shows the effectiveness of the design.

The main issue related to this kind of approach concerns the actual implementation of a metamaterial exhibiting the needed Lorentz dispersion at optical frequencies. However, as we have already shown in [11], it is possible to synthesize such engineered dispersive materials through a pile of alternating stacked disks of plasmonic materials and regular dielectrics. Choosing the right combination of materials and the proper thicknesses for the disks, it is possible to synthesize the desired profile of the material dispersion, in order to match the needed values of the permittivities of the two cylindrical shells at the two frequencies.

An example of the actual material implementation, related to the design of Figure 15, is reported in Figure 16. The cylindrical object made of silica is surrounded by two material shells. 


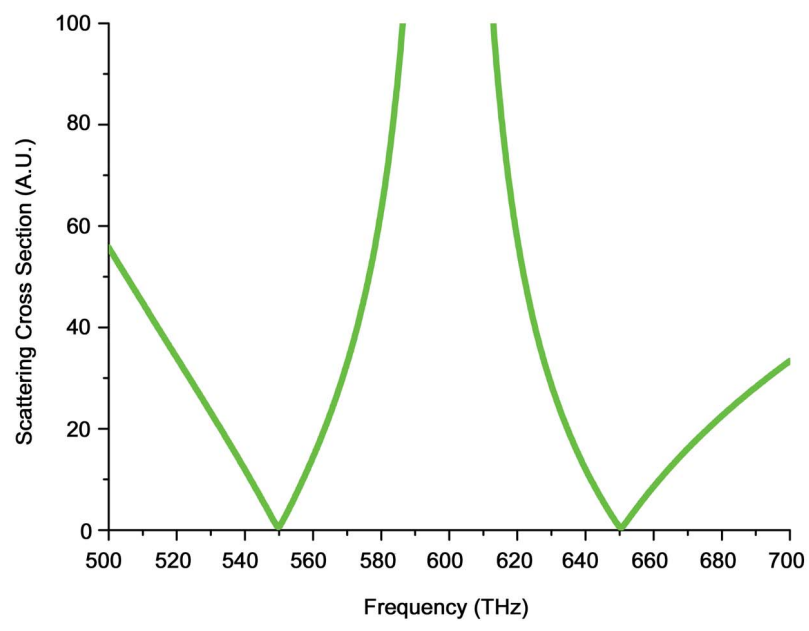

FIG. 15 Total scattering cross-section of a circular cylinder covered with a double layered shell designed to match the scattering cancellation condition at two distinct frequencies $f_{1}=555 \mathrm{THz}$ and $f_{2}=650 \mathrm{THz}$.
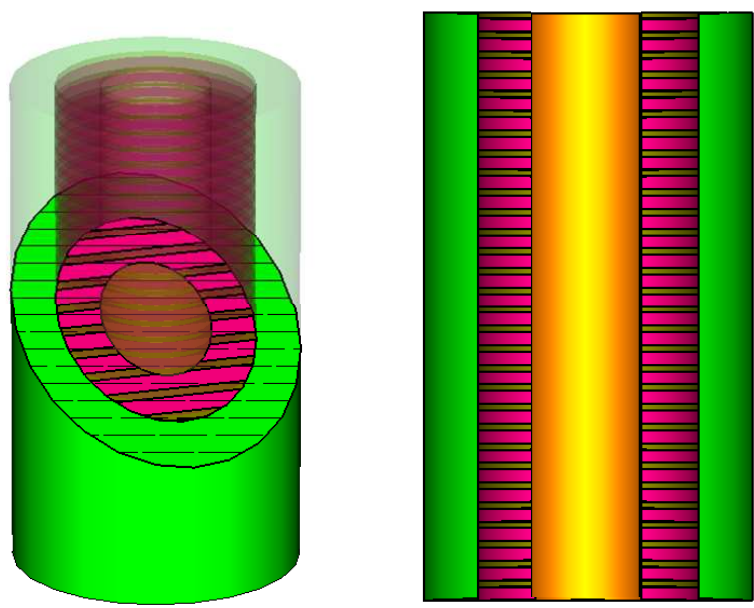

FIG. 16 Proposed setup to implement a double layered cover with the middle shell consisting of a Lorentz-like dispersive medium, synthesized through a pile of alternating stacked disks of a plasmonic material and a regular dielectric.

The outer shell is a homogeneous layer and is made of silver. The inner shell, instead, is made of alternating annular disks of a plasmonic material (e.g. silver) and a dielectric material at optical frequencies (e.g. silicon carbide or silica), such that the effective permittivity of the inhomogeneous material exhibits the required Lorentz-like dispersion.

\section{CONCLUSIONS}

In this paper, we have presented the design of cylindrical multi-layered electromagnetic cloaks, based on the scattering cancellation technique. We have demonstrated that a bilayered cylindrical shell is effectively capable of reducing the scattered width of a dielectric cylindrical object illuminated by a plane wave. We have investigated, then, the theoretical aspects related to the analysis of the proposed cloaking devices, giving, eventually, straightforward design rules. Moreover, we have extended the design approach to generic multilayered structures with more than two layers and we have proposed realistic setups of cloaking devices working at optical frequencies. Finally, we have validated our analysis and shown the effectiveness of the proposed design rules through a set of full-wave simulations confirming the predicted results. A possible application of multi-layered cloaking devices to multi-frequency operation has been also proposed and discussed.

\section{References}

[1] G. W. Milton and N. A. Nicorovici, "On the cloaking effects associated with anomalous localized resonances" P. Roy. Soc. A-Math. Phy. 462, 3027-3059 (2006).

[2] J. B. Pendry, D. Schurig, and D. R. Smith, "Controlling Electromagnetic Fields" Science 312, 1780 (2006).

[3] D. Schurig, J. J. Mock, B. J. Justice, S. A. Cummer, J. B. Pendry, A. F. Starr, and D. R. Smith, "Metamaterial Electromagnetic Cloak at microwave frequencies" Science 314, 977 (2006).

[4] U. Leonhardt, "Optical conformal mapping" Science 312, 1777-1780 (2006).

[5] W. Cai, U. K. Chettiar, A. V. Kildishev, and V. M. Shalaev, "Optical cloaking with metamaterials" Nat. Photonics 1, 224-227 (2007).

[6] Y. Luo, J. Zhang, B. Wu, and C. Hongsheng, "Interaction of an electromagnetic wave with a cone-shaped invisibility cloak and polarization rotator" Phys. Rev. B 78, 125108 (2008).

[7] A. Alù and N. Engheta, "Achieving transparency with plasmonic and metamaterial coatings" Phys. Rev. E 72, 016623 (2005).

[8] A. Alù and N. Engheta, "Plasmonic Materials in Transparency and Cloaking Problems: Mechanism, Robustness, and Physical Insights" Opt. Express 15, 3318 (2007).

[9] A. Alù and N. Engheta, "Cloaking and transparency for collections of particles with metamaterial and plasmonic covers" 0pt. Express 15, 7578 (2007).

[10] A. Alù and N. Engheta, "Multifrequency Optical Invisibility Cloak with Layered Plasmonic Shells" Phys. Rev. Lett. 100, 113901 (2008).

[11] F. Bilotti, S. Tricarico, and L. Vegni, Optical cloaking with metamaterials (Proc. of the EOS Topical Meeting on Nanophotonics, Photonic Crystals and Metamaterials 2008).

[12] J. A. Stratton, Electromagnetic Theory (McGraw-Hill Comp., New York and London, 1941).

[13] M. G. Silveirinha, A. Alù, and N. Engheta, "Parallel Plate Metamaterials for Cloaking Structures" Phys. Rev. E 75, 036603 (2007).

[14] H. E. Bussey and J. H. Richmond, "Scattering by a Lossy Dielectric Circular Cylindrical Multilayer, Numerical Values" IEEE T. Antenn. Propag. 23, 723-725 (1975)

[15] C. A. Balanis, Advanced Electromagnetic Engineering (John Wiley at Sons Comp., Hoboken, 1989). 